\title{
TAGUNG
}

\section{Konsolidierungskurs nach der Krise - Strategien für Europa}

\author{
Alexander Kalb und Marc-Daniel Moessinger*
}

Vom 24. bis 25. März 2011 veranstaltete der Arbeitskreis Europäische Integration e.V. gemeinsam mit dem Arbeitskreis Politische Ökonomie, der Universität Freiburg, dem Walter Eucken Institut sowie dem Zentrum für Europäische Wirtschaftsforschung (ZEW) eine Konferenz zum Thema „Konsolidierungskurs nach der Krise - Strategien für Europa“. Vor dem Hintergrund der infolge der Wirtschafts- und Finanzkrise dramatisch angestiegenen Haushaltsdefizite der EU-Mitgliedstaaten beschäftigte sich diese Konferenz mit der Analyse der aktuellen finanzpolitischen Entwicklungen, ihren Konsequenzen sowie möglicher Konsolidierungsstrategien für die EU-Mitgliedstaaten.

Die Konferenz wurde von Friedrich Heinemann eröffnet, der noch einmal auf die Wirtschafts- und Finanzkrise und insbesondere auf die aktuelle Krise des Euros einging. Vor diesem Hintergrund sei es notwendig, dass die verantwortlichen Politiker der Mitgliedstaaten der Eurozone (Euro-Staaten) gemeinsam Strategien ausarbeiten, die zur Stabilisierung des Euros sowie zu einer langfristig nachhaltigen Fiskalpolitik, die darauf abzielt übermäßige Staatsverschuldung zurückzuführen, in den EuroStaaten beitragen. Die nachfolgenden Beiträge widmeten sich genau diesen Themenfeldern.

Konsolidierung der öffentlichen Finanzen aus Sicht der Regierungen

Uwe Wagschal bezog sich in seinem Vortrag auf die Zeit vor Ausbruch der Wirtschafts- und

\section{„Post-Crisis Fiscal Consolidation Strategies for Europe 6}

Freiburg im Breisgau, 24.-25. März 2011

Welcomeadress

Friedrich HEINEMANN, Centre for European Economic Research, Mannheim

\section{The Governance of Consolidation}

The pre-crisis perspective: When do governments consolidate? A quantitative analysis of 23 OECD countries (1980-2005)

Uwe WAGSCHAL, University of Freiburg

The Euro Crisis from a constitutional economic perspective

Jan VOßWINKEL, Centrum für Europäische Politik, Freiburg

From First-Release to Ex-Post Fiscal Data: Exploring the sources of Revision Errors in the EU Roel BEETSMA, University of Amsterdam

Political Economy and Constitutional Consolidation of Public Debt

Does the Broad Public Want to Consolidate Public Debt? - The Role of Fairness and of Policy Credibility Helmut STIX, Economic Studies Division, Oesterreichische Nationalbank, Wien

The Debt Brake: Business Cycle and Welfare Consequences of Germany's New Fiscal Policy Rule Nikolai STÄHLER, Zentrale Öffentliche Finanzen, Deutsche Bundesbank, Frankfurt

Debt Brakes and Deficit Limits: On the Constitutional Economics of Intergenerational Justice

Bernhard NEUMÄRKER and Stephan WOLF, University of Freiburg

\section{Economic Effects of Public Debt}

Comparing good and bad borrowing

Martin PALDAM, School of Economics and

Management, Aarhus University

Budget Deficit Spillover Effects in the Euro Area Shafik HEBOUS, Goethe University Frankfurt

* Dr. Alexander Kalb, Senior-Researcher, Forschungsbereich Unternehmensbesteuerung und Öffentliche Finanzwirtschaft, Zentrum für Europäische Wirtschaftsforschung (ZEW), Mannheim.

Marc-Daniel Moessinger, Wissenschaftlicher Mitarbeiter, Forschungsbereich Unternehmensbesteuerung und Öffentliche Finanzwirtschaft, Zentrum für Europäische Wirtschaftsforschung (ZEW), Mannheim. 
Finanzkrise und analysierte die Konsolidierungsbemühungen von Regierungen. Im Zentrum der durchgeführten Untersuchungen standen sowohl die Identifikation als auch die Untersuchung derjenigen Faktoren, die Konsolidierungsanstrengungen beeinflussen beziehungsweise maßgeblich zu einer erfolgreichen Konsolidierung beitragen. Ebenfalls beantwortete er Fragen nach dem optimalen Zeitpunkt von Konsolidierungsanstrengungen und nach der optimalen Konsolidierungsstrategie. Aufbauend auf der Darstellung verschiedener Messkonzepte zur Definition von Konsolidierungsanstrengungen wurden die Ergebnisse einer quantitativen Analyse vorgestellt. Hierbei wurde deutlich, dass vor allem Nachfragefaktoren, wie zum Beispiel die Erhöhung der Arbeitslosenquote oder ein steigender relativer Anteil älterer Mitbürger an der Gesamtbevölkerung Konsolidierungsanstrengungen negativ beeinflussen. Demgegenüber erleichtere eine Erhöhung der Wachstumsrate mögliche Konsolidierungsbemühungen erheblich. Ebenfalls ginge eine höhere Staatsverschuldung mit einer größeren Wahrscheinlichkeit zur Budgetkonsolidierung einher. Mit Bezug auf eine nachhaltige Konsolidierungsstrategie erläuterte Wagschal den Vorzug von Ausgabenkürzungen gegenüber Einnahmeerhöhungen. Des Weiteren sollten Konsolidierungsbemühungen möglichst rasch nach Amtsübernahme begonnen werden, um die positiven Effekte der eigenen Regierungsarbeit noch in der laufenden Legislaturperiode nutzen zu können.

Jan Voßwinkel analysierte in seinem Beitrag die expliziten und impliziten konstitutionellen Veränderungen, die sich aus den Rettungsmaßnahmen in Verbindung mit der Schuldenkrise der Europäischen Union ergeben haben. Voßwinkel erläuterte die bereits vor der Krise bestehende Unglaubwürdigkeit des Stabilitäts- und Wachstumspaktes, der durch das Verhalten Deutschlands und Frankreichs im Jahre 2003 stark beschädigt worden sei. Ausgehend von einer verfassungsgemäß unabhängigen Europäischen Zentralbank und der Einführung der Europäischen Finanzstabilisierungsfazilität (EFSF), sei es durch die kri-

\section{Consolidation Strategies in the Euro Area}

Have the euro area and EU economic governance worked? Just the facts

Livio STRACCA, European Central Bank, Frankfurt

Towards expenditure rules and fiscal sanity in the euro area

Sebastian HAUPTMEIER, European Central Bank, Frankfurt

\section{Keynote Speech}

Do Budgetary Institutions Mitigate Political Influences on Fiscal Policy? New Empirical Evidence for the $E U$

Jakob DE HAAN, University of Groningen

On the Relation between Fiscal and Financial Crisis

Fiscal Rules and Risk Premia: National and Subnational Evidence

Marc-Daniel MOESSINGER, Centre for European Economic Research, Mannheim

Credit Ratings and the Euro Area sovereign debt crisis

António AFONSO, European Central Bank and Technical University of Lisbon

\section{Fiscal Policy in Crisis}

Automatic Stabilizers and Economic Crisis: US vs. Europe

Mathias DOLLS, University of Cologne

Fiscal policy over the business cycle in Germany - a tale in three acts

Christoph PRIESMEIER, Deutsche Bundesbank, Frankfurt

\section{Keynote Speech}

Public Debt: What you see is less than you have Bernd RAFFELHÜSCHEN, University of Freiburg

senbedingten Hilfsmaßnahmen $\mathrm{zu}$ einer Aushöhlung der ursprünglich verfolgten Ziele der Europäischen Wirtschafts- und Währungsunion gekommen. Durch die von den Staats- und Regierungschefs getroffenen Entscheidungen, hoch verschuldeten Mitgliedstaaten beizustehen, sowie der für die Europäische Zentralbank - wenn auch nur übergangsweise angedachten - geschaffenen Möglichkeit, nationale Anleihen am Markt aufzukaufen, sei die vertraglich festgelegte ,no bailout'-Klausel implizit gefallen. Der Wechsel der Europäischen Union hin zu einer Transferunion sei somit vorbestimmt, insbesondere, da für die Mitgliedstaaten bereits vor 
der Wirtschaftskrise ein erheblicher Vertrauensverlust in die Regelungen des Stabilitätsund Wachstumspaktes bestanden hätte. Ein möglicher Lösungsmechanismus der aufgezeigten Problematik könnte jedoch die Einführung nationaler Fiskalregeln darstellen, die das Problem übermäßiger Staatsverschuldung auf nationalem Weg eindämmen könnten.

Roel Beetsma stellte das Problem der Abweichungen zwischen erstveröffentlichten und ex post veröffentlichten Ist-Daten zu Haushaltsgrößen in den Mittelpunkt seines Vortrages und analysierte Bestimmungsfaktoren dieser Abweichungen. Von entscheidender Bedeutung sei das Problem, dass erstveröffentlichte Daten im laufenden Haushaltsjahr als Planungsgrundlage für die folgenden Jahre verwendet werden. Demnach beruhe auch die fiskalische Überwachung auf diesen Daten, was bei großen Abweichungen zum nachträglich feststellbaren Ist-Zustand zu erheblichen Verzerrungen führen könne. Vor allem Regierungen könnten demnach ihren direkten oder indirekten Einfluss auf die Veröffentlichung erster Ist-Daten zur laufenden Rechnungsperiode (aus-)nutzen, um überoptimistische Prognosen abzugeben. Zur Verdeutlichung dieser Problematik stellte Beetsma den bereits systematisch zu nennenden Unterschied der Datenqualität verschiedener Staaten der Europäischen Union gegenüber. Entsprechend könne bei bestimmten Staaten von einem nahezu konstanten Korrekturfaktor ausgegangen werden. Auf Grundlage einer empirischen Untersuchung bestehe somit durch die Verbesserung der fiskalischen Institutionen, wie zum Beispiel durch strengere nationale Fiskalregeln, der Anwendung beziehungsweise Durchsetzung der mittelfristigen Haushaltsziele der Europäischen Union sowie der Stärkung der Transparenz bei der Haushaltsaufstellung, ein erhebliches Potenzial, die Datenqualität erstveröffentlichter IstDaten zu steigern. Die Ergebnisse würden ebenso die Forderung der Europäischen Kommission nach Minimalstandards bei der Haus- haltsaufstellung unterstützen und des Weiteren für eine strengere Überwachung durch die Kommission plädieren.

\section{Konsolidierung der öffentlichen Finanzen mithilfe von Fiskalregeln}

Helmut Stix ging in seiner Studie der Frage nach, wann und unter welchen Voraussetzungen die Wähler Konsolidierungen der öffentlichen Haushalte unterstützen beziehungsweise ablehnen. Hierfür zog Stix die Ergebnisse einer Umfrage heran, die im Jahr 2010 in Österreich durchgeführt wurde. Für diese Umfrage wurden 2.000 Bürgern Österreichs per Zufallsauswahl unter anderem Fragen über ihre Präferenzen bezüglich der Konsolidierung der öffentlichen Finanzen gestellt. Die Ergebnisse dieser Umfrage wurden anschließend einer empirischen Analyse unterzogen. Dabei stellte sich heraus, dass sich zwar eine Mehrheit der Wähler für eine Konsolidierung der Haushalte aussprach; die Präferenzen hinsichtlich der Konsolidierung der Haushalte variierten jedoch erheblich unter den Bürgern. So sprachen sich beispielsweise ältere Menschen weniger oft für eine Konsolidierung der Haushalte aus als jüngere. Zudem scheint auch die intergenerationelle Gerechtigkeit eine bedeutende Rolle zu spielen: So plädierten Befragte mit eigenen Kindern deutlich häufiger für die Konsolidierung der öffentlichen Haushalte.

Nikolai Stähler referierte in seinem Vortrag über die deutsche Schuldenbremse, die auf Bundesebene ab 2016 beziehungsweise auf Länderebene ab 2020 verbindlich eingeführt wird. Konkret konzentrierte sich Stähler in seinem Vortrag auf die Auswirkungen der Schuldenbremse im Hinblick auf den Verlauf der Konjunkturzyklen sowie auf die daraus resultierenden Wohlfahrtseffekte. Ein zentraler Teil der Analyse erstreckte sich dabei auf den Vergleich der Schuldenbremse mit den automatischen Stabilisatoren. Im Rahmen eines neu-keynesianischen DSGE-Modellansatzes $^{1}$ zeigte Stähler, dass sowohl von der

1 DSGE steht für Dynamic Stochastic General Equilibrium. Bei DSGE-Modellen handelt es sich um mikroökonomisch fundierte Modelle zur Erklärung dynamischer makroökonomischer Zusammenhänge. 
Schuldenbremse als auch von automatischen Stabilisatoren ähnliche Effekte auf den Verlauf von Konjunkturzyklen ausgehen. Bezüglich der Wohlfahrtswirkungen argumentierte Stähler, dass automatische Stabilisatoren vergleichsweise geringere Wohlfahrtsverluste bewirken. Daraus schlussfolgerte er, dass Fiskalregeln, deren Wirkungsweise denen von automatischen Stabilisatoren ähneln, grundsätzlich der Vorzug gegeben werden sollte.

Der Vortrag von Bernhard Neumärker und Stephan Wolf beleuchtete hingegen die Einführung numerischer Fiskalregeln vor dem Hintergrund der intergenerationellen Gerechtigkeit. So argumentierten beide Referenten, dass die Limitierung der Defizite beziehungsweise Neuverschuldung eines Staates schon im Hinblick auf die Belastungen der zukünftigen Generationen zwingend notwendig sei. Neumärker und Wolf legten in ihrem Vortrag dar, dass die gängige Argumentation für die Aufnahme neuer Schulden eines Staates aus keynesianischer Sicht nicht gerechtfertigt sei. Sie begründeten diese These damit, dass die keynesianische Theorie das Streben der Politiker nach einer Wiederwahl unberücksichtigt lasse. Genau dies führe aber dazu, dass die Politiker Ausgaben hauptsächlich über Schulden und nicht etwa über zusätzliche Steuereinnahmen finanzieren würden (,Political Business Cycles"), was wiederum zulasten der zukünftigen Generationen erfolge. Aus diesem Grund sei die Einführung etwa der deutschen Schuldenbremse durchaus gerechtfertigt. Neumärker und Wolf bemängelten indes die ungenaue Definition des Begriffs der Investitionen bei der , alten' Schuldenbremse nach Art. 115 Grundgesetz. Als Kritikpunkte für die ,neue' Schuldenbremse nach Art. 109 Abs. 3 Grundgesetz führten sie die Manipulierbarkeit der Konjunkturkomponente auf. Zudem sei in der neuen Schuldenbremse kein Mechanismus enthalten, der zu einem Abbau der bereits akkumulierten Schulden führe.

\section{Ökonomische Auswirkungen öffentlicher Ver-} schuldung

Der Vortrag von Martin Paldam konzentrierte sich auf die Verschuldung von Entwicklungsländern und deren Auswirkungen auf das ökonomische Wachstum. Mithilfe eines Datensatzes über 59 Entwicklungsländer und einem Zeitraum von 15 Jahren untersuchte Paldam in einem ersten Schritt den Zusammenhang zwischen dem Verschuldungsgrad eines Landes und seiner ökonomischen Wachstumsrate. Die Ergebnisse der Regressionsanalyse deuteten - wie erwartet - auf einen negativen $\mathrm{Zu}$ sammenhang hin. Da die Verschuldung jedoch nur einen geringen Teil der Variation der Wachstumsraten erklären konnte, unterzog Paldam die Länder einer weiteren Analyse, indem er jeweils zwei Länder - eines mit einer hohen und eines mit einer niedrigen Wachstumsrate jedoch mit vergleichbaren Verschuldungsstrukturen - gegenüberstellte. Hieraus konnte er interessante Schlussfolgerungen ziehen: So zeigten Entwicklungsländer, die durch einen höheren Grad sowohl an ökonomischer als auch politischer Freiheit gekennzeichnet sind, höhere Wachstumsraten. Als weitere wichtige Determinanten einer positiven Wachstumsentwicklung identifizierte Paldam den Entwicklungsgrad eines Landes, die Verfügbarkeit von natürlichen Ressourcen sowie die Ausprägung demokratischer Strukturen.

Mit Spill-over-Effekten von fiskalischen Schocks eines Landes auf andere Staaten beschäftigte sich der Vortrag von Shafik Hebous. Unter Anwendung eines sogenannten Global Vector Autoregression (GVAR) Modells untersuchte Hebous, ob sich Defizitschocks in Mitgliedstaaten der Europäischen Union auch auf die Outputs anderer Mitgliedstaaten auswirken. Die Ergebnisse eines bilateralen Vergleichs der Staaten konnten jedoch keinen eindeutigen Trend aufdecken: So können von Defizitschocks entweder positive oder negative Output-Effekte auf den jeweiligen Partnerstaat ausgehen - je nachdem welche Staaten für den Vergleich herangezogen 
werden. Bezüglich der Output-Effekte merkte Hebous indes an, dass diese sehr häufig nicht signifikant und somit vernachlässigbar seien. In einer weiteren Analyse konnte Hebous jedoch einen signifikant positiven Einfluss eines eurozonenweiten Defizitschocks auf die Outputs aller Mitgliedstaaten identifizieren, woraus der Autor der Studie schlussfolgerte, dass die Fiskalpolitik in der Eurozone gemeinsam koordiniert werden sollte.

\section{Konsolidierungsstrategien in der Eurozone}

Livio Stracca untersuchte in seinem Beitrag, inwiefern die beiden Säulen der Europäischen Union, der Stabilitäts- und Wachstumspakt sowie die Lissabon-Strategie, einen wirksamen Beitrag hinsichtlich der von ihnen verfolgten Ziele geleistet haben. Zur empirischen Analyse verwendete Stracca Daten sowohl der Europäischen Union als auch Daten einer Vergleichsgruppe von OECD-Staaten. ${ }^{2}$ Im Ergebnis sei festzustellen, dass beide, der Stabilitäts- und Wachstumspakt und auch die Vorgaben der Lissabon-Strategie die Fiskalpolitik und die ökonomische Leistungsfähigkeit in den Mitgliedstaaten der Europäischen Union nicht wesentlich verbessert haben. Von einem optimistischen Standpunkt aus betrachtet könne der fehlende Einfluss des Stabilitäts- und Wachstumspaktes jedoch auch positiv gedeutet werden: Immerhin seien Befürchtungen hinsichtlich eines negativen Einflusses einer Währungsunion auf die nationale Fiskalpolitik nicht eingetreten. Demnach könnten sich positive und negative Einflüsse gerade neutralisiert haben. Um zukünftig eine verbesserte fiskalische Situation sowie eine höhere ökonomische Leistungsfähigkeit zu erreichen, müssten die beiden Säulen zwingend weiter gestärkt werden. Ein wesentliches Element könnten in diesem Kontext strengere nationale Fiskalregeln sein.

Sebastian Hauptmeier fokussierte in seinem Vortrag auf die Ausgabenpolitik innerhalb der Europäischen Union, um darauf aufbauend die unterschiedlichen fiskalischen Entwicklungen zu untersuchen. Während die Entwicklung $\mathrm{zu}$ einer nachhaltigen Fiskalpolitik entsprechend den Vorgaben des Stabilitätsund Wachstumspaktes im Vorkrisenzeitraum einen positiven Verlauf nahm, habe die Wirtschafts- und Finanzkrise zu einer erheblichen Abweichung geführt. Treibender Faktor sei in diesem Zusammenhang die Ausgabenentwicklung in den Mitgliedstaaten gewesen. Ziel der durchgeführten Studie sei es somit, die Ausgaben- und Schuldenentwicklung innerhalb der Mitgliedstaaten unter der Annahme einer hypothetisch angewendeten Ausgabenregelung $\mathrm{zu}$ untersuchen. Unter Verwendung von Echtzeitdaten, das heißt Daten die in den entsprechenden Jahren als Planungsgrößen in den Mitgliedstaaten vorlagen, wurde diese Entwicklung modelliert. Eine hypothetische Ausgabenregelung, die sich an der Entwicklung des Bruttoinlandsproduktes orientiert, hätte somit wesentlich zu einer vernünftigeren Fiskalpolitik beigetragen. Entsprechend sei der institutionelle Rahmen Europas sowohl auf nationaler als auch auf supranationaler Ebene durch eine effektivere Ausgabenkontrolle beziehungsweise -überwachung zu stärken.

Vermindern Budgetprozessregeln den Einfluss der Fiskalpolitik?

Jakob De Haan befasste sich in seinem Vortrag mit der Frage, inwiefern der Einfluss von Budgetinstitutionen auf die Fiskalpolitik durch politische Fragmentierung beeinflusst wird. Aufgrund der Tatsache, dass Politiker in Demokratien Anreizen ausgesetzt sind, die Steuerbasis übermäßig auszunutzen, ergibt sich die Notwendigkeit Budgetinstitutionen einzurichten. Diese können die für die Bevölkerung insgesamt negativen Anreize vermindern. Hierzu hätten sich in der Literatur zwei unterschiedliche Modelle entwickelt. Zum einen der Delegationsansatz, bei dem besondere (Vor-)Rechte an einen starken Finanzminister übertragen werden, und zum anderen der

2 Organisation for Economic Co-operation and Development. 
Vertragsansatz, der auf einer für das gesamte Kabinett verbindlichen ex ante-Regel beruht. Anstatt nur den Einfluss der beiden jeweiligen Lösungen auf das Haushaltsergebnis zu untersuchen, sei es ebenfalls von Bedeutung, die Auswirkungen politischer Fragementierung auf die Wirksamkeit der jeweiligen Budgetinstitutionen zu analysieren. Zur Messung der politischen Fragmentierung könnten hierzu verschiedene Indikatoren, wie zum Beispiel die Anzahl der Parteien oder Minister einer Regierung oder ein Maß für die ideologische Distanz innerhalb der Regierung (beispielsweise ob eine Koalition aus einem MitteLinks-Bündnis oder zwei linken Parteien besteht), verwendet werden.

Auf Grundlage der durchgeführten empirischen Untersuchungen zeige sich, dass sowohl der Delegationsansatz als auch die Vertragslösung gerade im Falle einer großen ideologischen Fragmentierung innerhalb der Regierung einen bedeutenden Effekt auf die Nachhaltigkeit der Fiskalpolitik habe. Beide Budgetinstitutionen sind demnach in der Lage, den negativen Einfluss der politischen Fragmentierung zu verringern. Im Gegensatz dazu sei der Einfluss beider institutioneller Lösungsmechanismen weder durch die Anzahl der Parteien noch durch die Anzahl der Minister beeinflusst. Dies widerspräche zuvor veröffentlichten Studien, die die Wirksamkeit der beiden Budgetinstitutionen auch in $\mathrm{Ab}$ hängigkeit der politischen Fragmentierung sehen.

Zusammenhang zwischen fiskalischer und Finanzkrise

Marc-Daniel Moessinger befasste sich mit der Verbindung nationaler Fiskalregeln und deren Einfluss auf die Höhe der Risikoaufschläge nationaler Schuldverschreibungen. Aufbauend auf der vergleichenden Darstellung der Renditeunterschiede nationaler Anleihen zur deutschen Bundesanleihe vor und nach Ausbruch der Wirtschafts- und Finanzkrise stellte er die Vorgehensweise sowie die empirischen Ergebnisse sowohl für die natio- nale Ebene im europäischen Kontext als auch für die subnationale Ebene anhand kantonaler Daten der Schweiz vor. In beiden Untersuchungen wurde jeweils ein Index zur Abbildung der Stärke nationaler beziehungsweise kantonaler Fiskalregeln verwendet und dessen Einfluss auf Renditeunterschiede zu einer risikolosen deutschen beziehungsweise Schweizer Bundesanleihe gemessen. Staaten sowie Kantone, die im Betrachtungszeitraum Fiskalregeln eingesetzt hatten, hätten demnach im Durchschnitt geringere Risikoaufschläge im Vergleich zu Jurisdiktionen mit keinen oder nur schwachen Fiskalregeln zu tragen. Besonders die Robustheit der Ergebnisse im nationalen als auch subnationalen Kontext sei hierbei von entscheidender Bedeutung und untermauere den positiven Einfluss von Fiskalregeln auf die Finanzierungskosten eines Staates oder Kantons. Da der gewählte Betrachtungszeitraum die Krisenperiode explizit ausschließe, ergäbe sich aufgrund der durch die Krise gestiegenen Sensibilität der Märkte ein für die Nachkrisenperiode gestiegenes Potenzial dieses Budgetinstruments.

António Afonso erläuterte in seinem Vortrag den Einfluss von Ratingentscheidungen sowie Ratingprognosen der drei größten Ratingagenturen Standard \& Poor's, Moody's und Fitch Ratings auf die Renditeunterschiede nationaler Staatsanleihen in der Europäischen Union. Durch die empirische Auswertung tagesaktueller Daten über Ratingeinstufungen sowie Renditeunterschiede sei ein signifikanter Einfluss sowohl von Ratingverbesserungen als auch Ratingverschlechterungen auf Renditeunterschiede festzustellen. Diese unterschieden sich jedoch dahingehend, dass der Effekt einer Ratingherabstufung zu einer stärkeren Reaktion der Renditeunterschiede führt. Ebenfalls erläuterte Afonso, dass seinen Untersuchungen zufolge kein Antizipationseffekt vorläge. Dementsprechend würden Ratingveränderungen - und hier vor allem Ratingherabstufungen - nicht von den Anleihemärkten antizipiert. Jedoch sei eine zweiseitige Kausalität zwischen Ratingeinstufungen und Renditeaufschlägen signifikant 
nachzuweisen: Je höher die Renditeaufschläge sind, desto schlechter sei die Ratingeinstufung und vice versa. Die Schlussfolgerung der Ergebnisse impliziere demnach, dass die von Ratingänderungen überraschten Marktteilnehmer entweder Fundamentaldaten zu wenig beachten oder aber Ratingagenturen aufgrund der spezifischen Vernetzung über einen erheblichen Informationsvorteil verfügen. Jedoch bestände für souveräne Staaten die Möglichkeit, Ratingveränderungen durch eine nachhaltige Fiskalpolitik vorzubeugen und damit die Refinanzierungskosten durch eine Verhinderung einer Ratingherabstufung zu vermindern.

\section{Fiskalpolitik in der Krise}

Der Vortrag von Mathias Dolls beschäftigte sich mit der Rolle von automatischen Stabilisatoren in Krisenzeiten im Kontext der Mitgliedstaaten der Europäischen Union sowie den Vereinigten Staaten. Mithilfe der Mikrosimulationsprogramme EUROMOD und TAXSIM untersuchte Dolls, inwiefern die Steuer- und Transfersysteme der Europäischen Union einerseits und der Vereinigten Staaten andererseits in der gegenwärtigen Wirtschafts- und Finanzkrise als automatische Stabilisatoren gewirkt haben. Die von Dolls präsentierten Ergebnisse deuteten darauf hin, dass die automatischen Stabilisatoren in den Mitgliedstaaten der Europäischen Union circa 38 Prozent eines proportionalen Einkommensschocks abfedern können; die Abfederung in den Vereinigten Staaten beträgt hingegen nur 32 Prozent. Ähnlich verhält es sich auch mit Schocks am Arbeitsmarkt: Während in den Mitgliedstaaten der Europäischen Union 30 Prozent der Schocks durch automatische Stabilisatoren aufgefangen werden können, sind es in den Vereinigten Staaten nur 20 Prozent. Dolls merkte jedoch an, dass es innerhalb der Europäischen Union deutliche Unterschiede gebe: So sind die Wirkungen der automatischen Stabilisatoren in den Staaten Ost- und Südeuropas deutlich weniger ausgeprägt als die der Staaten Zentral- und Nordeuropas.
Das ,Timing' diskretionärer fiskalpolitischer Maßnahmen von Regierungen war Gegenstand des Vortrags von Christoph Priesmeier. Konkret ging Priesmeier der Frage nach, ob die Fiskalpolitik der vergangenen 40 Jahre in Deutschland eher durch eine anti-, pro- oder azyklische Fiskalpolitik charakterisiert war. Zudem versuchte Priesmeier mit seinem Beitrag zu klären, ob sich das Timing der Reaktionen der Bundesregierung auf OutputSchocks über den genannten Zeitablauf eher verbessert oder verschlechtert hat. Unter Anwendung spezieller vektorautoregressiver Modelle konnte Priesmeier vier unterschiedlich charakterisierte Perioden identifizieren: Zwischen 1976 und 1980 beziehungsweise 1980 und 1984 wirkten die von der Bundesregierung ergriffenen Maßnahmen - als Reaktionen auf Output-Schocks - sehr stark antizyklisch, wobei dieser antizyklische Effekt zwischen 1980 und 1984 im Vergleich zur Vorperiode deutlich schwächer ausfiel. In den beiden Folgeperioden (1984 bis 1991 und 1991 bis 2008) schwächten sich die antizyklischen Effekte der diskretionären Maßnahmen der Bundesregierung immer weiter ab. Priesmeier schlussfolgerte daraus, dass die Reaktionen der Bundesregierung auf OutputSchocks in den beiden zuletzt genannten Perioden eher eine azyklische Wirkung entfaltet hätten.

\section{Das wahre Ausmaß der öffentlichen Verschul-} dung

Im Abschlussvortrag dieser Konferenz referierte Bernd Raffelhüschen über das Ausmaß der impliziten Verschuldung und die daraus resultierenden finanziellen Belastungen für die nachkommenden Generationen in Deutschland. Der Ausgangspunkt seines Vortrags war die Feststellung, dass die explizite oder ,sichtbare“ Verschuldung aller öffentlichen Haushalte in Deutschland momentan bei circa 2 Billionen Euro oder 79 Prozent des Bruttoinlandsprodukts liege. Er argumentierte weiter, dass dies nur ein (Bruch-)Teil der Gesamtverschuldung Deutschlands sei, da dieser Wert nicht die impliziten oder zukünftigen 
Verbindlichkeiten des Staates enthalte. Implizite Verbindlichkeiten würden in vier großen Bereichen anfallen: bei den Sozialversicherungssystemen, den Pensionen staatlicher Beamter, im Gesundheitsbereich sowie bei der Langzeitpflege älterer Menschen.

Bezüglich der Sozialversicherungssysteme führe der negative demografische Wandel, der sich in den nächsten Jahrzehnten in Deutschland immer weiter vollziehen wird, zu einer Finanzierungslücke im umlagefinanzierten Rentenversicherungssystem, die zu den expliziten Verbindlichkeiten des Staates hinzugezählt werden müsse. Anschließend kam Raffelhüschen auf die Problematik der Beamtenpensionen zu sprechen. Vor allem dann, wenn die sogenannte Babyboomer-Generation in den Ruhestand eintreten werde, werden die Pensionslasten, die überwiegend aus Steuergeldern finanziert werden, deutlich ansteigen. Dies führe zu einer weiteren Finanzierungslücke, die bei den expliziten Verbindlichkeiten berücksichtigt werden müsse. Im Gesundheitsbereich führen vor allem die angestiegene Lebenserwartung sowie die starke Zunahme der Kosten im Gesundheitswesen aufgrund des immer weiter voranschreitenden technologischen Fortschritts zu einer Lücke zwischen tatsächlich anfallenden und tatsächlich geleisteten Zahlungen. Diese Finanzierungslücke werde durch den negativen demografischen Wandel zusätzlich vergrößert, da immer weniger junge Menschen für immer mehr ältere Menschen aufkommen müssten. Ähnliche Argumente brachte Raffelhüschen auch für die Entstehung einer Finanzierungslücke im Bereich der Langzeitpflege älterer Menschen vor.

Raffelhüschen schlussfolgerte daraus, dass unter Berücksichtigung all dieser impliziten Verbindlichkeiten - die Staatsschuld in Deutschland insgesamt bei ungefähr 6,5 Billionen oder 289 Prozent des Bruttoinlandsprodukts (optimistisches Szenario) beziehungsweise 11 Billionen Euro oder 479 Prozent des Bruttoinlandsprodukts (realistisches Szenario) liegen würde. Ein internationaler Vergleich zeigte schließlich auf, dass nicht nur Deutschland Tragfähigkeitsprobleme bezüglich der öffentlichen Finanzen aufweist; auch Länder wie etwa Frankreich oder das Vereinigte Königreich hätten neben den expliziten sehr hohe implizite Verbindlichkeiten. 